\title{
On the Stability of $f$-Maximal Spacelike Hypersurfaces in Weighted Generalized Robertson-Walker Spacetimes
} by

\author{
Eudes L. DE LIMA, Henrique F. DE LIMA and Fábio R. DOS SANTOS
}

Presented by Tadeusz IWANIEC

Summary. Our purpose in this paper is to study the stability of $f$-maximal spacelike hypersurfaces immersed in a weighted generalized Robertson-Walker spacetime $-I \times{ }_{\rho} M_{f}^{n}$, where $M_{f}^{n}$ is a weighted Riemannian manifold endowed with a weight function $f$. In this setting, we obtain sufficient conditions to guarantee that an $f$-maximal hypersurface be $L_{f}$-stable, where $L_{f}$ stands for the weighted Jacobi operator.

1. Introduction. Let $\left(\bar{M}^{n+1},\langle\rangle,\right)$ be an orientable $(n+1)$-dimensional Lorentzian manifold endowed with a timelike vector field $V$ and let $f$ : $\bar{M}^{n+1} \rightarrow \mathbb{R}$ be a smooth function. The weighted Lorentzian manifold $\bar{M}_{f}^{n+1}$ associated with $\bar{M}^{n+1}$ and $f$ is the triple $\left(\bar{M}^{n+1},\langle\rangle,, e^{-f} d \bar{M}\right)$, where $d \bar{M}$ denotes the standard volume element of $\bar{M}^{n+1}$ induced by the metric $\langle$,$\rangle .$ We will refer to the function $f$ as being the weight function associated to $\bar{M}_{f}^{n+1}$. In this setting, an important tensor is the Bakry-Émery Ricci tensor $\overline{\operatorname{Ric}}_{f}$, a natural generalization of the Ricci tensor $\overline{\operatorname{Ric}}$ of $\bar{M}_{f}^{n+1}$ defined by

$$
\overline{\operatorname{Ric}}_{f}=\overline{\operatorname{Ric}}+\overline{\operatorname{Hess}} f,
$$

where $\overline{\text { Hess }} f$ is the Hessian of $f$ on $\bar{M}_{f}^{n+1}$.

Appearing naturally in the study of self-shrinkers, Ricci solitons, harmonic heat flows and many other subjects in differential geometry, weighted

2010 Mathematics Subject Classification: Primary 53C42; Secondary 53B30, 53C50.

Key words and phrases: generalized Robertson-Walker spacetimes, weighted manifolds, $f$-maximal spacelike hypersurfaces, stable hypersurfaces.

Received 5 May 2016; revised 9 August 2016.

Published online 30 September 2016. 
manifolds proved to be important nontrivial generalizations of Riemannian manifolds and they are objects of extensive ongoing investigation. For a brief overview of results in this area, we refer the articles of Morgan [M] and Wei-Wylie WW].

In this context, we consider a (connected) spacelike hypersurface $\psi$ : $\Sigma^{n} \rightarrow \bar{M}_{f}^{n+1}$ immersed in a weighted Lorentzian manifold $\bar{M}_{f}^{n+1}$, that is, the metric induced on $\Sigma^{n}$ via $\psi$ is a Riemannian metric. As usual, we also denote by $\langle$,$\rangle the metric of \Sigma^{n}$ induced via $\psi$. Since $V$ is a globally defined timelike vector field on $\bar{M}_{f}^{n+1}$, there exists a unique unitary timelike normal vector field $N$ globally defined on $\Sigma^{n}$ which is in the same timeorientation of $V$, that is, $\langle V, N\rangle<0$. We will refer to this normal timelike vector field $N$ as being the future-pointing Gauss map of $\Sigma^{n}$. Throughout this work, $N$ will always denote the future-pointing Gauss map of a spacelike hypersurface $\psi: \Sigma^{n} \rightarrow \bar{M}_{f}^{n+1}$. On the other hand, we note that the weight function $f$ induces a weighted measure $e^{-f} d \sigma$ on $\Sigma^{n}$, where $d \sigma$ denotes the standard volume element of $\Sigma^{n}$ with respect to the induced metric from the ambient space $\bar{M}_{f}^{n+1}$. So, we have an induced weighted Riemannian manifold $\left(\Sigma^{n},\langle\rangle,, e^{-f} d \sigma\right)$.

The $f$-divergence operator on $\Sigma^{n}$ is defined by

$$
\operatorname{Div}_{f}(X)=e^{f} \operatorname{Div}\left(e^{-f} X\right),
$$

where $X$ is a tangent vector field on $\Sigma^{n}$ and Div denotes the standard divergence operator of $\Sigma^{n}$. From $(1.2)$ we can define the $f$-Laplacian of $\Sigma^{n}$ by

$$
\Delta_{f} u=\operatorname{Div}_{f}(\nabla u)=\Delta u-\langle\nabla f, \nabla u\rangle,
$$

where $u$ is a smooth function on $\Sigma^{n}, \Delta$ denotes the Laplacian induced by Div and $\nabla$ stands for the Levi-Civita connection of $\Sigma^{n}$ induced from the Levi-Civita connection $\bar{\nabla}$ on the ambient space $\bar{M}_{f}^{n+1}$.

Following Gromov $[\mathrm{G}$, the weighted mean curvature, or simply $f$-mean curvature, $H_{f}$ of $\Sigma^{n}$ is defined by

$$
n H_{f}=n H-\langle\bar{\nabla} f, N\rangle,
$$

where $H=-\frac{1}{n} \operatorname{tr}(A)$ denotes the standard mean curvature of $\Sigma^{n}$ and $A$ : $\mathfrak{X}(\Sigma) \rightarrow \mathfrak{X}(\Sigma)$, given by $A X=-\bar{\nabla}_{X} N$, is the shape operator of $\Sigma^{n}$ with respect to its future-pointing Gauss map $N$. So, a spacelike hypersurface $\psi: \Sigma^{n} \rightarrow \bar{M}_{f}^{n+1}$ immersed in a weighted Lorentzian manifold $\bar{M}_{f}^{n+1}$ is called an $f$-maximal hypersurface if its $f$-mean curvature $H_{f}$ vanishes identically or, equivalently, if its mean curvature $H$ satisfies

$$
n H=\langle\bar{\nabla} f, N\rangle \text {. }
$$


Stability questions concerning constant mean curvature compact hypersurfaces in Riemannian space forms began with Barbosa-do Carmo [BC], and Barbosa-do Carmo-Eschenburg [BCE]. In the former paper, the authors introduced the notion of stability and proved that spheres are the only stable critical points for the area functional, for volume preserving variations. In the setting of spacelike hypersurfaces in Lorentz manifold, Barbosa-Oliker [BO] proved that constant mean curvature spacelike hypersurfaces are critical points for volume preserving variations. Moreover, by computing the second variation formula they showed that constant mean curvature embedded spheres in the de Sitter space $\mathbb{S}_{1}^{n+1}$ maximize the area functional for such variations. Later on, Barros-Brasil-Caminha [BBC] classified strongly stable spacelike hypersurfaces with constant mean curvature immersed into so-called generalized Robertson-Walker spacetimes $-I \times{ }_{\rho} M^{n}$, that is, Lorentzian warped products with 1-dimensional negative definite base $I \subset \mathbb{R}$, Riemannian fiber $M^{n}$ and warping function $\rho: I \rightarrow \mathbb{R}$. Assuming a certain convexity condition on the warping function, they showed that a closed strongly stable spacelike hypersurface immersed with constant mean curvature in $-I \times{ }_{\rho} M^{n}$ is either maximal or a spacelike slice $\left\{t_{0}\right\} \times M^{n}$.

Proceeding in this direction, our aim is to investigate the $L_{f}$-stability of $f$-maximal spacelike hypersurfaces immersed in a weighted generalized Robertson-Walker spacetime $-I \times{ }_{\rho} M_{f}^{n}$, where $L_{f}$ stands for the weighted Jacobi operator defined by

$$
L_{f}=\Delta_{f}-\left(|A|^{2}+\overline{\operatorname{Ric}}_{f}(N, N)\right) .
$$

Here, motivated by a splitting theorem due to Case [C], we will suppose that the weight function $f$ does not depend on the parameter $t \in I$.

This manuscript is organized as follows. In Section 2 we compute the first and second variation formulas for a spacelike hypersurface in a weighted Lorentzian manifold (see Lemmas 2.1 and 2.2). Next, in Section 3 we establish an $L_{f}$-stability criterion (see Lemma 3.2 and, finally, we apply it to determine when a $f$-maximal spacelike hypersurface immersed in a weighted generalized Robertson-Walker spacetime $I \times{ }_{\rho} M_{f}^{n}$ is $L_{f}$-stable (see Theorem 3.3.

2. Preliminaries. In what follows, $\bar{M}^{n+1}$ denotes an $(n+1)$-dimensional Lorentzian manifold endowed with a timelike vector field $V, f: \bar{M}^{n+1} \rightarrow \mathbb{R}$ a smooth function and $\bar{M}_{f}^{n+1}$ the weighted Lorentzian manifold associated with $\bar{M}^{n+1}$ and $f$. If $\psi: \Sigma^{n} \rightarrow \bar{M}_{f}^{n+1}$ is a (connected) spacelike hypersurface in $\bar{M}_{f}^{n+1}$, then $\Sigma^{n}$ is automatically orientable (see [O, p. 189]) and we can choose a globally defined unit timelike normal vector field $N$ on $\Sigma^{n}$ which is in the same time-orientation as $V$, that is, $\langle V, N\rangle<0$. Moreover, 
as already mentioned, we have an induced weighted Riemannian manifold $\left(\Sigma^{n},\langle\rangle,, e^{-f} d \sigma\right)$.

The weighted area functional of $\Sigma^{n}$ is naturally defined by

$$
\operatorname{vol}_{f}(\Sigma)=\int_{\Sigma} e^{-f} d \sigma .
$$

The first and second variation formulas for the weighted area functional are well known in the case of hypersurfaces immersed in Riemannian spaces (see, for instanse, [CMZ]). In the present context, we have not found their proof in the current literature. So, for the sake of completeness, we will deduce them here.

For this, let $F: \Sigma^{n} \times(-\varepsilon, \varepsilon) \rightarrow \bar{M}^{n+1}$ be a variation of $\Sigma^{n}$ with compact support and fixed boundary, that is, $F(\cdot, t): \Sigma^{n} \rightarrow \bar{M}^{n+1}, t \in(-\varepsilon, \varepsilon)$, is a spacelike immersion and

(i) $F=$ Id outside a compact subset of $\Sigma^{n}$;

(ii) $F(x, 0)=\psi(x)$ for all $x \in \Sigma^{n}$;

(iii) $F(x, t)=x$ for all $x \in \partial \Sigma$.

The vector field $F_{t}=\left.\frac{\partial F}{\partial t}\right|_{t=0}$ restricted to $\Sigma^{n}$ is called the variational vector field of the variation $F$. We note that

$$
F_{t}=F_{t}^{\top}-\left\langle F_{t}, N\right\rangle N,
$$

where ()$^{\top}$ denotes the tangential component of a vector field in $\mathfrak{X}(\bar{M})$ along $\Sigma^{n}$. The variation $F$ is called normal when $F_{t}^{\top}=0$ on $\Sigma^{n}$.

LEMMA 2.1. Let $\bar{M}_{f}^{n+1}$ be a weighted Lorentzian manifold and let $\psi$ : $\Sigma^{n} \rightarrow \bar{M}_{f}^{n+1}$ be a spacelike hypersurface. If $F: \Sigma^{n} \times(-\varepsilon, \varepsilon) \rightarrow \bar{M}^{n+1}$ is a variation of $\Sigma^{n}$ with compact support and fixed boundary, then

$$
\left.\frac{d}{d t} \operatorname{vol}_{f}(F(\Sigma, t))\right|_{t=0}=n \int_{\Sigma} H_{f} \varphi e^{-f} d \sigma .
$$

where $F_{t}=F_{t}^{\top}+\varphi N$ (thus $\varphi=-\left\langle F_{t}, N\right\rangle$ ). In particular, $\Sigma^{n}$ is $f$-maximal if, and only if, $\left.\frac{d}{d t} \operatorname{vol}_{f}(F(\Sigma, t))\right|_{t=0}=0$ for every variation $F$ with compact support and fixed boundary.

Proof. Let $\left(x_{1}, \ldots, x_{n}\right)$ be a local coordinate system on $\Sigma^{n}$. Then $\left(F \circ x_{1}, \ldots, F \circ x_{n}\right)$ is a local coordinate system on $\Sigma_{t}=F(\Sigma, t)$. Denote $F_{x_{i}}(t)=d F\left(\frac{\partial}{\partial x_{i}}\right)$ and consider

$$
g_{i j}(t)=\left\langle F_{x_{i}}(t), F_{x_{j}}(t)\right\rangle \quad \text { and } \quad v(t)=\frac{\sqrt{\operatorname{det}\left(g_{i j}(t)\right)}}{\sqrt{\operatorname{det}\left(g_{i j}(0)\right)}} .
$$

Note that $v(t)$ is well defined and independent of the choice of a local coordinate system on $\Sigma^{n}$. Furthermore, denoting by $d \sigma_{t}$ the standard volume 
element of $\Sigma_{t}$ with respect to the metric induced from the ambient space, we have

$$
\operatorname{vol}_{f}(F(\Sigma, t))=\int_{\Sigma_{t}} e^{-f(p, t)} d \sigma_{t}=\int_{\Sigma} v(t) e^{-f(p, t)} d \sigma,
$$

where $f(p, t)=f(F(p, t))$. Differentiating this, we obtain

$$
\left.\frac{d}{d t} \operatorname{vol}_{f}(F(\Sigma, t))\right|_{t=0}=\left.\int_{\Sigma} \frac{d}{d t}\left(v(t) e^{-f(x, t)}\right)\right|_{t=0} d \sigma .
$$

Now, to evaluate $\left.\frac{d}{d t}\left(v(t) e^{-f(p, t)}\right)\right|_{t=0}$ at some point $p \in \Sigma^{n}$, we may choose a local coordinate system which is orthonormal at $p$. It is well known that $\left.\frac{d}{d t} v(t)\right|_{t=0}=\operatorname{Div} F_{t}^{T}+n H \varphi$. Hence, we get

$$
\begin{aligned}
\frac{d}{d t}\left(v(t) e^{-f(x, t)}\right) & \left.\right|_{t=0} \\
& =\left.e^{-f(p, 0)} \frac{d}{d t} v(t)\right|_{t=0}-\left.v(0) e^{-f(p, 0)} \frac{d}{d t} f(F(p, t))\right|_{t=0} \\
& =e^{-f}\left(\operatorname{Div}_{t} F_{t}^{T}+n H \varphi\right)-e^{-f}\left\langle\bar{\nabla} f, F_{t}\right\rangle \\
& =e^{-f}\left(\operatorname{Div}_{f} F_{t}^{T}+n H_{f} \varphi\right) .
\end{aligned}
$$

Therefore, taking into account the weighted version of the divergence theorem (see [CR, Lemma 2.2]), we can use (2.1) and (2.2) to conclude the proof.

Our aim is to study $f$-maximal spacelike hypersurfaces $\psi: \Sigma^{n} \rightarrow \bar{M}^{n+1}$ which maximize the weighted area functional for every normal variation of $\Sigma^{n}$ with compact support and fixed boundary. Hence, in order to examine when an $f$-maximal spacelike hypersurface is actually a maximum of weighted area functional, one certainly needs to study the second variation $\left.\frac{d^{2}}{d t^{2}} \operatorname{vol}_{f}(F(\Sigma, t))\right|_{t=0}$. In the next result, we compute the second variation formula of the weighted area functional.

LEMMA 2.2. Let $\bar{M}_{f}^{n+1}$ be a weighted Lorentzian manifold and let $\psi$ : $\Sigma^{n} \rightarrow \bar{M}_{f}^{n+1}$ be an $f$-maximal spacelike hypersurface. If $F: \Sigma^{n} \times(-\varepsilon, \varepsilon) \rightarrow$ $\bar{M}^{n+1}$ is a normal variation of $\Sigma^{n}$ with compact support and fixed boundary, then

$$
\left.\frac{d^{2}}{d t^{2}} \operatorname{vol}_{f}(F(\Sigma, t))\right|_{t=0}=\int_{\Sigma} \varphi\left(\Delta_{f} \varphi-\left(|A|^{2}+\overline{\operatorname{Ric}}_{f}(N, N)\right) \varphi\right) e^{-f} d \sigma,
$$

where $F_{t}=\varphi N\left(\right.$ thus $\left.\varphi=-\left\langle F_{t}, N\right\rangle\right)$.

Proof. In what follows, we keep the notation established in the proof of Lemma 2.1. We have

$$
\left.\frac{d^{2}}{d t^{2}} \operatorname{vol}_{f}(F(\Sigma, t))\right|_{t=0}=\left.\int_{\Sigma} \frac{d^{2}}{d t^{2}}\left(v(t) e^{-f(x, t)}\right)\right|_{t=0} d \sigma
$$


It is well known that

$$
\left.\frac{d^{2}}{d t^{2}} v(t)\right|_{t=0}=\operatorname{Div}\left(\bar{\nabla}_{F_{t}} F_{t}\right)+n^{2} \varphi^{2} H^{2}-|\nabla \varphi|^{2}-\left(|A|^{2}+\overline{\operatorname{Ric}}(N, N)\right) \varphi^{2} .
$$

On the other hand, using the fact that $\Sigma^{n}$ is $f$-maximal jointly with (2.4), with a straightforward computation we obtain

$$
\begin{aligned}
\left.\frac{d^{2}}{d t^{2}}\left(v(t) e^{-f(x, t)}\right)\right|_{t=0} & \\
= & e^{-f}\left(\left.\frac{d^{2}}{d t^{2}} v(t)\right|_{t=0}-\left.\left\langle\bar{\nabla} f, F_{t}\right\rangle \frac{d}{d t} v(t)\right|_{t=0}-\left.v(0) \frac{d}{d t}\left\langle\bar{\nabla} f, F_{t}\right\rangle\right|_{t=0}\right) \\
& +\left.\frac{d}{d t} e^{-f(x, t)}\right|_{t=0}\left(\left.\frac{d}{d t} v(t)\right|_{t=0}-v(0)\left\langle\bar{\nabla} f, F_{t}\right\rangle\right) \\
= & e^{-f}\left(\operatorname{Div}_{f}\left(\bar{\nabla}_{F_{t}} F_{t}\right)-|\nabla \varphi|^{2}-\left(|A|^{2}+\overline{\operatorname{Ric}_{f}}(N, N)\right) \varphi^{2}\right) .
\end{aligned}
$$

Therefore, using once more the weighted divergence theorem and the fact that $\Sigma^{n}$ is $f$-maximal we conclude our proof.

3. Stability of $f$-maximal spacelike hypersurfaces. It follows from Lemma 2.2 that the second variation formula for the weighted area functional depends only on $\varphi \in C_{0}^{\infty}$. So, the following definition makes sense.

Definition 3.1. Let $\bar{M}_{f}^{n+1}$ be a weighted Lorentzian manifold and let $\psi: \Sigma^{n} \rightarrow \bar{M}_{f}^{n+1}$ be an $f$-maximal spacelike hypersurface. We say that $\Sigma^{n}$ is $L_{f}$-stable if, for any compactly supported smooth function $\varphi \in C_{0}^{\infty}(\Sigma)$,

$$
\left.\frac{d^{2}}{d t^{2}} \operatorname{vol}_{f}(\Sigma)\right|_{t=0}=\int_{\Sigma} \varphi L_{f} \varphi e^{-f} d \sigma \leq 0,
$$

where the weighted Jacobi operator $L_{f}$ is defined by (1.5).

To prove our main theorem, we will also need the following auxiliary result.

LEMma 3.2. Let $\bar{M}_{f}^{n+1}$ be a weighted Lorentzian manifold and let $\psi$ : $\Sigma^{n} \rightarrow \bar{M}_{f}^{n+1}$ be an $f$-maximal spacelike hypersurface. If there exists a positive smooth function $u \in C^{\infty}(\Sigma)$ such that $L_{f} u \leq 0$, then $\Sigma^{n}$ is $L_{f}$ stable.

Proof. Assume that such a $u$ exists and take $\varphi \in C_{0}^{\infty}(\Sigma)$. Then, we can choose $\eta \in C_{0}^{\infty}(\Sigma)$ satisfying $\varphi=\eta u$. Hence, from 1.5 we have 


$$
\begin{aligned}
\int_{\Sigma} \varphi L_{f} & \varphi e^{-f} d \sigma=\int_{\Sigma} \eta u L_{f}(\eta u) e^{-f} d \sigma \\
& =\int_{\Sigma}\left[\eta^{2} u L_{f} u+\eta u^{2} \Delta \eta+2 \eta u\langle\nabla u, \nabla \eta\rangle-\eta u^{2}\langle\nabla \eta, \nabla f\rangle\right] e^{-f} d \sigma \\
& \leq \int_{\Sigma}\left[\eta u^{2} \Delta \eta+2 \eta u\langle\nabla u, \nabla \eta\rangle-\eta u^{2}\langle\nabla \eta, \nabla f\rangle\right] e^{-f} d \sigma \\
& =\int_{\Sigma}\left[\eta u^{2} \Delta \eta+\frac{1}{2}\left\langle\nabla u^{2}, \nabla \eta^{2}\right\rangle-\eta u^{2}\langle\nabla \eta, \nabla f\rangle\right] e^{-f} d \sigma .
\end{aligned}
$$

On the other hand, it is not difficult to verify that

$$
\begin{aligned}
\operatorname{Div}\left(u^{2} \nabla \eta^{2}\right) & =\left\langle\nabla u^{2}, \nabla \eta^{2}\right\rangle+u^{2} \Delta \eta^{2} \\
& =\left\langle\nabla u^{2}, \nabla \eta^{2}\right\rangle+2 \eta u^{2} \Delta \eta+2 u^{2}|\nabla \eta|^{2} .
\end{aligned}
$$

Therefore, using once more the weighted version of the divergence theorem, from (3.1) and (3.2) we get

$$
\begin{aligned}
\int_{\Sigma} \varphi L_{f} \varphi e^{-f} d \sigma & \leq \int_{\Sigma}\left[\frac{1}{2} \operatorname{Div}\left(u^{2} \nabla \eta^{2}\right)-\eta u^{2}\langle\nabla \eta, \nabla f\rangle-u^{2}|\nabla \eta|^{2}\right] e^{-f} d \sigma \\
& =\int_{\Sigma}\left[\frac{1}{2} \operatorname{Div}_{f}\left(u^{2} \nabla \eta^{2}\right)-u^{2}|\nabla \eta|^{2}\right] e^{-f} d \sigma \\
& \leq-\int_{\Sigma} u^{2}|\nabla \eta|^{2} e^{-f} d \sigma \leq 0,
\end{aligned}
$$

and consequently $\Sigma^{n}$ is $L_{f}$-stable.

Now, let $M^{n}$ be a (connected) complete $n$-dimensional Riemannian manifold, $I \subset \mathbb{R}$ an open interval in $\mathbb{R}$ and $\rho: I \rightarrow \mathbb{R}$ a positive smooth function on $I$. We will denote by $\bar{M}^{n+1}=-I \times{ }_{\rho} M^{n}$ the product manifold $I \times M^{n}$ endowed with the Lorentzian metric

$$
\langle,\rangle=-\pi_{I}^{*}\left(d t^{2}\right)+\left(\rho \circ \pi_{I}\right)^{2} \pi_{M}^{*}\left(\langle,\rangle_{M}\right),
$$

where $\pi_{I}$ and $\pi_{M}$ denote the canonical projections from $I \times M^{n}$ onto each factor, $\langle,\rangle_{M}$ is the Riemannian metric on the fiber $M^{n}$, and $I$ is endowed with the metric $-d t^{2}$. The Lorentzian manifold $\bar{M}^{n+1}$ is called a Lorentzian warped product with base $I$, fiber $M^{n}$ and warping function $\rho$.

When $M^{n}$ has constant sectional curvature, the warped product $\bar{M}^{n+1}=$ $-I \times{ }_{\rho} M^{n}$ has been known in the literature as a Robertson-Walker (RW) spacetime, due to the fact that, for $n=3$, it is an exact solution of Einstein's field equations (see [0, Chapter 12]). After [ARS], the warped product $\bar{M}^{n+1}=-I \times{ }_{\rho} M^{n}$ has usually been referred to as a generalized RobertsonWalker (GRW) spacetime, and we will stick to this terminology along this paper. 
Let $\bar{M}^{n+1}=-I \times{ }_{\rho} M^{n}$ be a GRW spacetime and $f: \bar{M}^{n+1} \rightarrow \mathbb{R}$ a smooth function on $\bar{M}^{n+1}$. It follows from a splitting theorem due to Case (see [C, Theorem 1.2]) that if a weighted GRW spacetime $\bar{M}_{f}^{n+1}$ with bounded weight function $f$ is such that $\overline{\operatorname{Ric}}_{f}(T, T) \geq 0$ for all timelike vector fields $T$, then $f$ must be constant along $I$. Motivated by this result, here we will consider weighted GRW spacetimes $\bar{M}_{f}^{n+1}$ whose weight function $f$ does not depend on $t \in I$, that is, $\left\langle\bar{\nabla} f, \partial_{t}\right\rangle=0$; for simplicity, we will denote them by $\bar{M}_{f}^{n+1}=-I \times_{\rho} M_{f}^{n}$.

Recall that, since $\partial_{t}$ is a globally defined timelike vector field on $\bar{M}_{f}^{n+1}$, there exists a unique unitary timelike normal vector field $N$ globally defined on $\Sigma^{n}$ which is in the same time-orientation as $\partial_{t}$, i.e. $\left\langle N, \partial_{t}\right\rangle \leq-1$. In this context, we will consider one particular smooth function, namely, the angle function $\Theta=\left\langle N, \partial_{t}\right\rangle$, where $\partial_{t}$ stands for the unitary vector field which determines on $\bar{M}^{n+1}=-I \times{ }_{\rho} M^{n}$ a codimension one foliation by totally umbilical slices $\{t\} \times M$.

Now, we will state and prove our main result concerning $L_{f}$-stability of spacelike hypersurfaces in a weighted GRW spacetime.

TheOREM 3.3. Let $\psi: \Sigma^{n} \rightarrow \bar{M}_{f}^{n+1}$ be an $f$-maximal spacelike hypersurface immersed into a weighted GRW spacetime $\bar{M}_{f}^{n+1}=-I \times{ }_{\rho} M_{f}^{n}$.

(a) If $\rho^{\prime \prime} \leq 0$ on $\Sigma^{n}$, then $\Sigma^{n}$ is $L_{f}$-stable.

(b) If $\Sigma^{n}$ is compact and $\rho^{\prime \prime} \geq 0$ on $\Sigma^{n}$, then $\Sigma^{n}$ is $L_{f}$-stable if and only if $\rho^{\prime \prime}=0$ on $\Sigma^{n}$.

(c) If $\Sigma^{n}$ is compact and $\rho^{\prime \prime}>0$ on $\Sigma^{n}$, then $\Sigma^{n}$ cannot be $L_{f}$-stable.

Proof. First, we will prove (a). For this, let us consider on $\Sigma^{n}$ the negative function $\tilde{\Theta}=\rho \Theta$. Since $\rho \partial_{t}$ is a conformal vector field in $\bar{M}_{f}^{n+1}$ with $\bar{\nabla}_{X} \rho \partial_{t}=\rho^{\prime} X$ for every $X \in \mathfrak{X}(\Sigma)$, we can see that

$$
\begin{aligned}
X(\tilde{\Theta}) & =X\left\langle N, \rho \partial_{t}\right\rangle=\left\langle\bar{\nabla}_{X} N, \rho \partial_{t}\right\rangle+\left\langle N, \bar{\nabla}_{X} \rho \partial_{t}\right\rangle \\
& =-\left\langle A X, \rho \partial_{t}\right\rangle=-\left\langle X, A\left(\rho \partial_{t}^{\top}\right)\right\rangle .
\end{aligned}
$$

So, from the last equation we obtain $\nabla \tilde{\Theta}=-\rho A\left(\partial_{t}^{\top}\right)$, and from [CL, Proposition 2.1],

$$
\Delta \tilde{\Theta}=n \rho \partial_{t}^{\top}(H)+n \rho^{\prime} H-n N\left(p^{\prime}\right)+\left(|A|^{2}+\overline{\operatorname{Ric}}(N, N)\right) \tilde{\Theta} .
$$

On the other hand, taking into account our restriction on the weight function, from (1.4) we get

$$
\begin{aligned}
n \partial_{t}^{\top}(H) & =\partial_{t}^{\top}\langle\bar{\nabla} f, N\rangle \\
& =\left\langle\bar{\nabla}_{\partial_{t}} \bar{\nabla} f, N\right\rangle+\Theta\left\langle\bar{\nabla}_{N} \bar{\nabla} f, N\right\rangle-\left\langle\bar{\nabla} f, A\left(\partial_{t}^{\top}\right)\right\rangle \\
& =\overline{\operatorname{Hess}} f\left(N, \partial_{t}\right)+\Theta \overline{\operatorname{Hess}} f(N, N)+\rho^{-1}\langle\nabla f, \nabla \tilde{\Theta}\rangle .
\end{aligned}
$$


Moreover, it is not difficult to verify that

$$
\overline{\operatorname{Hess}} f\left(N, \partial_{t}\right)=-\rho^{-1} \rho^{\prime}\langle\bar{\nabla} f, N\rangle=-n \rho^{-1} \rho^{\prime} H .
$$

It follows from (3.4)-(3.6) that

$$
\Delta \tilde{\Theta}=\langle\nabla f, \nabla \tilde{\Theta}\rangle-n N\left(\rho^{\prime}\right)+\left(|A|^{2}+\overline{\operatorname{Ric}}_{f}(N, N)\right) \tilde{\Theta} .
$$

Thus, from (1.3) we obtain

$$
\begin{aligned}
\Delta_{f} \tilde{\Theta} & =\Delta \tilde{\Theta}-\langle\nabla f, \nabla \tilde{\Theta}\rangle \\
& =-n N\left(\rho^{\prime}\right)+\left(|A|^{2}+\overline{\operatorname{Ric}}_{f}(N, N)\right) \tilde{\Theta} .
\end{aligned}
$$

Now, writing $N=N^{*}-\Theta \partial_{t}$, where $N^{*}$ denotes the orthogonal projection of $N$ onto the fiber $M^{n}$, we find that

$$
N\left(\rho^{\prime}\right)=-\Theta \partial_{t}\left(\rho^{\prime}\right)=-\frac{\rho^{\prime \prime}}{\rho} \tilde{\Theta} .
$$

Hence, we conclude that

$$
L_{f} \tilde{\Theta}=n \frac{\rho^{\prime \prime}}{\rho} \tilde{\Theta} .
$$

Therefore, we conclude that $\Sigma^{n}$ is $L_{f}$-stable.

Now, let us consider (b). In this case, we have $C_{0}^{\infty}(\Sigma)=C^{\infty}(\Sigma)$. So, if $\Sigma^{n}$ is $L_{f}$-stable, we obtain

$$
0 \geq \int_{\Sigma} \tilde{\Theta} L_{f} \tilde{\Theta} e^{-f} d \sigma=\int_{\Sigma} n \frac{\rho^{\prime \prime}}{\rho} \tilde{\Theta}^{2} e^{-f} d \sigma \geq 0
$$

that is, $\rho^{\prime \prime}=0$ on $\Sigma^{n}$. The converse follows from item (a).

Finally, we prove (c). From the definition of $L_{f}$-stability we conclude that

$$
\int_{\Sigma} \tilde{\Theta} L_{f} \tilde{\Theta} e^{-f} d \sigma=\int_{\Sigma} n \frac{\rho^{\prime \prime}}{\rho} \tilde{\Theta}^{2} e^{-f} d \sigma>0
$$

Therefore, $\Sigma^{n}$ cannot be $L_{f}$-stable.

REMARK 3.4. Let $\bar{M}_{f}^{n+1}=-I \times_{\rho} M_{f}^{n}$ be a weighted GRW spacetime whose fiber $M^{n}$ is compact. In particular, the slices in $\bar{M}_{f}^{n+1}$ are also compact. Assume in addition that $\rho^{\prime \prime} \geq 0$ and that

$$
\Omega=\left\{t \in I: \rho^{\prime \prime}(t)=0\right\}
$$

is a set of isolated points. Then, for every $t \in \Omega$ such that $\rho^{\prime}(t)=0$, the $f$-maximal slice $\Sigma_{t}=\{t\} \times M^{n}$ is $L_{f}$-stable.

Acknowledgements. The second author is partially supported by CNPq, Brazil, grant 303977/2015-9. The authors would like to thank the referee for giving several valuable suggestions and comments which improved the paper. 


\section{References}

[ARS] L. J. Alías, A. Romero and M. Sánchez, Uniqueness of complete spacelike hypersurfaces of constant mean curvature in generalized Robertson-Walker spacetimes, Gen. Relat. Grav. 27 (1995), 71-84.

[BBC] A. Barros, A. Brasil and A. Caminha, Stability of spacelike hypersurfaces in foliated spacetimes, Differential Geom. Appl. 26 (2008) 357-365.

[BC] J. L. M. Barbosa and M. do Carmo, Stability of hypersurfaces with constant mean curvature, Math. Z. 185 (1984), 339-353.

[BCE] J. L. M. Barbosa, M. do Carmo and J. Eschenburg, Stability of hypersurfaces with constant mean curvature, Math. Z. 197 (1988), 123-138.

[BO] J. L. M. Barbosa and V. Oliker, Spacelike hypersurfaces with constant mean curvature in Lorentz space, Mat. Contemp. 4 (1993), 27-44.

[CL] A. Caminha and H. F. de Lima, Complete vertical graphs with constant mean curvature in semi-Riemannian warped products, Bull. Belg. Math. Soc. Simon Stevin 16 (2009), 91-105.

[CR] A. Cañete and C. Rosales, Compact stable hypersurfaces with free boundary in convex solid cones with homogeneous densities, Calc. Var. 51 (2014), 887-913.

[C] J. S. Case, Singularity theorems and the Lorentzian splitting theorem for the Bakry-Émery-Ricci tensor, J. Geom. Phys. 60 (2010), 477-490.

[CLS] M. P. Cavalcante, H. F. de Lima and M. S. Santos, New Calabi-Bernstein type results in weighted generalized Robertson-Walker spacetimes, Acta Math. Hungar. 142 (2015), 440-454.

[CMZ] X. Cheng, T. Mejia and D. Zhou, Stability and compactness for complete $f$ minimal surfaces, Trans. Amer. Math. Soc. 367 (2015), 4041-4059.

[G] M. Gromov, Isoperimetry of waists and concentration of maps, Geom. Funct. Anal. 13 (2003), 178-215.

[M] F. Morgan, Manifolds with density, Notices Amer. Math. Soc. 52 (2005), 853-858.

[O] B. O'Neill, Semi-Riemannian Geometry with Applications to Relativity, Academic Press, London, 1983.

[WW] G. Wei and W. Willie, Comparison geometry for the Bakry-Émery Ricci tensor, J. Differential Geom. 83 (2009), 377-405.

Eudes L. de Lima

Campus Pau dos Ferros

Universidade Federal Rural do Semi-Árido

59.900-000 Pau dos Ferros

Rio Grande do Norte, Brazil

E-mail: eudes.leite@ufersa.edu.br
Henrique F. de Lima, Fábio R. dos Santos

Departamento de Matemática

Universidade Federal de Campina Grande 58.429-970 Campina Grande, Paraíba, Brazil E-mail: henrique@mat.ufcg.edu.br fabio@dme.ufcg.br 